\section{Molecular attributes underlying central nervous system and systemic relapse in diffuse large B-cell lymphoma}

Refractory or relapsed diffuse large B-cell lymphoma (DLBCL) is challenging to treat in general and progression in the central nervous system (CNS) is associated with particularly dismal outcomes. Indeed, the median survival of patients diagnosed with CNS relapse is typically 2-4 months. ${ }^{1}$ The incidence of CNS relapse is $2-5 \%$ in unselected patient cohorts treated with modern immunochemotherapy, ${ }^{2,3}$ presenting a challenge for risk prediction. Individual risk factors for CNS relapse are well established, and include clinical parameters such as age, stage, elevated lactate dehydrogenase and performance status. ${ }^{1,4}$ Moreover, the involvement of certain extranodal sites such as uterus/testis or kidney/adrenal gland confers higher risk of CNS relapse. ${ }^{5}$ These individual clinical risk factors have been integrated into a risk score (CNS International Prognostic Index [CNS-IPI]). Molecular attributes such as double-hit translocation status, BCL2/MYC dual protein expression as well as the activated B-cell-like (ABC) subtype (or non-germinal center $B$ [GCB]), have also been associated with a higher risk of CNS relapse. ${ }^{6,7}$ However, the major shortcoming of available risk prediction models is their limited discriminative power. While the CNS-IPI classifies $12 \%$ of patients into the high-risk group, only $10 \%$ of patients within this high-risk group experience CNS relapse. ${ }^{3}$

Herein, to comprehensively assess molecular determinants of CNS relapse, we chose three distinct approaches. Firstly, we performed exome sequencing in paired tissue biopsies (peripheral and CNS parenchymal tumors) and reconstructed clonal phylogenies that underlie CNS invasion. Secondly, we assembled a dataset to assess the association of specific gene mutations with CNS relapse. Lastly, we performed gene expression and pathway analysis on an enriched cohort of diagnostic tissue samples from 222 patients with diverging clinical outcomes. An overview of all samples used in this study is shown in the Online Supplementary Figure S1.

In order to describe changes in clonal structure associated with CNS relapse, we used the PyClone computational tool to infer clonal structures in five paired CNS samples (Online Supplementary Table S1). Recurrent gene mutations included PIM1 $(\mathrm{n}=4)$, CD $44(\mathrm{n}=3)$, ETV6 $(\mathrm{n}=$ 3), MALAT1 $(\mathrm{n}=3)$, ARHGAP5 $(\mathrm{n}=2), C D 79 B(\mathrm{n}=2)$, CXCR4 $(\mathrm{n}=2)$ and RUNX1T1 $(\mathrm{n}=2)$. We observed striking clonal divergence in all five cases, with biopsies from the time of CNS invasion characterized by discrete sets of mutations (Figure 1). In general, the expanding subclones were not sampled at the time point of diagnosis, with the main exception being case LY_CNSrel_006. The number of mutations found in CNS-relapsing clones tended to be higher than the number of shared mutations, and higher than the number of mutations exclusively found at diagnosis (Online Supplementary Figure S2). The most frequently mutated gene was PIM1, with 22 exonic, intronic and untranslated region mutations identified in samples from four patients, in keeping with PIM1 being recognized as a known target of aberrant somatic hypermutation in DLBCL. ${ }^{8}$ These mutations were mostly subclonal (95\%), identified in either expanding (14 of 22 mutations, $64 \%$ ) or extinguished clones (seven of 22 mutations, $32 \%$ ). The ETS family member transcription factor ETV6 was found to be mutated in expanding clones in two cases, and in an ancestral clone in another case, with all mutations map- ping to the exon 1 donor splice site. Our findings document the existence of intratumoral heterogeneity that is associated with the emergence of subclones that are observed in the CNS. They also suggest that the disease leading to relapse has features that are distinct from the disease found at primary diagnosis.

Next, we asked whether a larger cohort of diagnostic samples could delineate gene mutations associated with either CNS or systemic relapse. We compiled mutation data from 223 diagnostic DLBCL samples, derived from several datasets of both population-based and clinical trial series. Information on 45 genes was available for 72 cases with CNS relapse, 62 cases with systemic relapse and 89 cases without relapse (Online Supplementary Table S2). The strongest signal for enrichment within cases with subsequent CNS relapse was found for MYD88, although this gene was almost as frequently mutated in cases with subsequent systemic relapse (Figure 2). On the other hand, several gene mutations appeared most robustly associated with systemic relapse, and were comparatively uncommon in cases with subsequent CNS relapse. This pattern was most striking for TP53, with mutations found in $45 \%$ of patients experiencing systemic relapse, in $20 \%$ of patients with no relapse, and in only $11 \%$ of patients with subsequent CNS relapse. Furthermore, we found that certain gene mutations were relatively depleted in CNS relapse cases, compared to cases with either systemic or no relapse. The most striking example was $K M T 2 D$, mutated in $22 \%$ of patients with CNS relapse, compared to $39 \%$ and $37 \%$ of patients with no relapse and systemic relapse, respectively.

Lastly, we asked whether specific pathways were enriched in diagnostic samples from patients who subsequently experienced either CNS or systemic relapse. We included a total of 222 patients with samples available for gene expression profiling: 50 patients with CNS relapse $(n=39)$ or CNS involvement documented at diagnosis $(\mathrm{n}=11), 64$ patients with systemic relapse and 108 patients without relapse (Online Supplementary Table S3). We performed gene set enrichment analysis to identify biological processes deregulated in the three risk groups (Online Supplementary Table S4). Interestingly, we observed a significant downregulation of pathways in ABC-DLBCL when comparing systemic versus no relapse, and an upregulation of pathways in GCBDLBCL (Figure 3A). Downregulation of pathways was also observed in CNS versus no relapse in ABC-DLBCL but not GCB-DLBCL. We observed that systemic relapse in GCB-DLBCL appeared to be driven by MYC, metabolic and protein translation signatures, to a higher degree than CNS relapse (Figure 3B). We assessed whether the double-hit signature ${ }^{9}$ allowed the delineation of varying risks of CNS and/or systemic relapse in our cohort. Overall, 39 of 90 classifiable GCB cases $(43 \%)$ were positive for a double-hit signature. The percentage of double-hit signature-postive-positive cases was similar between systemic (64\%) and CNS relapse $(58 \%)$, and lower in cases without relapse $\left(29 \%, \chi^{2}\right.$ test $P=0.008$, Online Supplementary Figure S3). These results link expression of the DHIT signature with CNS relapse, mirroring the increased risk of CNS relapse conferred by dual expression of MYC and BCL2. ${ }^{6} \mathrm{ABC}-\mathrm{DLBCL}$ cases with subsequent relapse were characterized by depletion of stromal signatures, an effect that was most pronounced in those ABC-DLBCL cases with systemic relapse. In summary, while cases with subsequent CNS relapse displayed similar pathway enrichment patterns to cases with subsequent systemic relapse, there are like- 
A
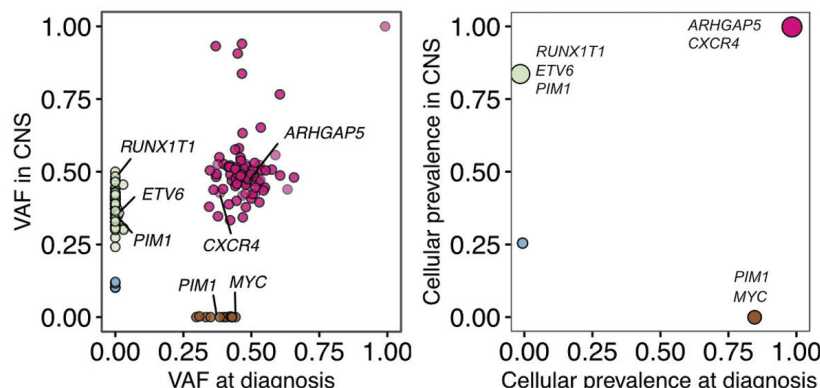

B

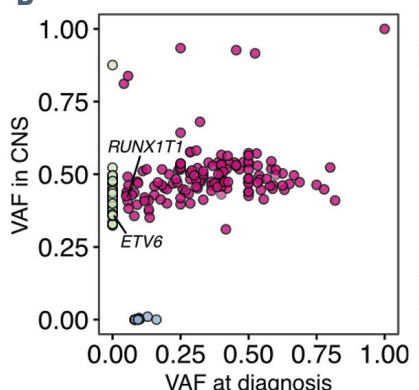

C
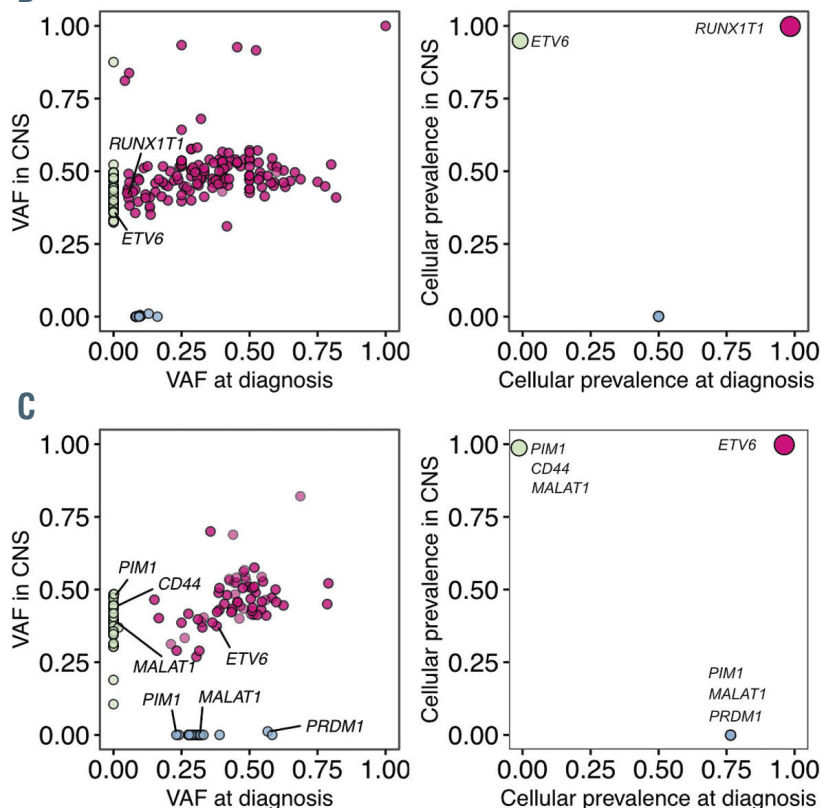

D

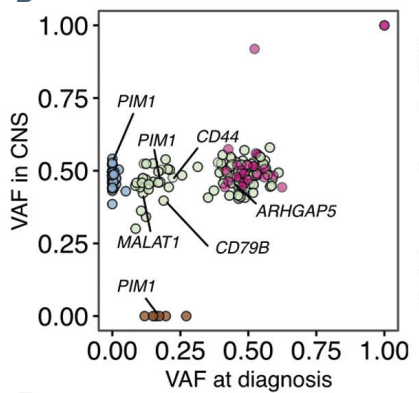

E

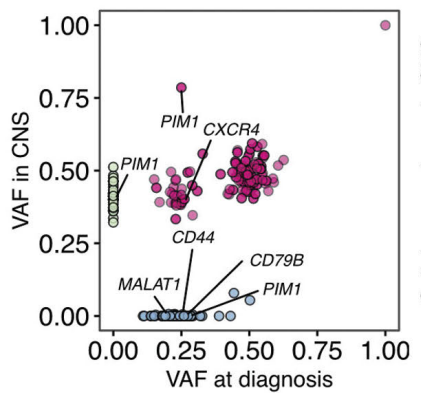

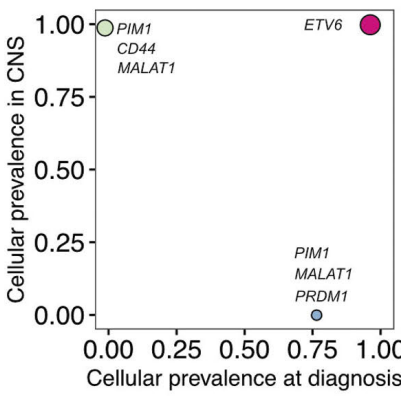
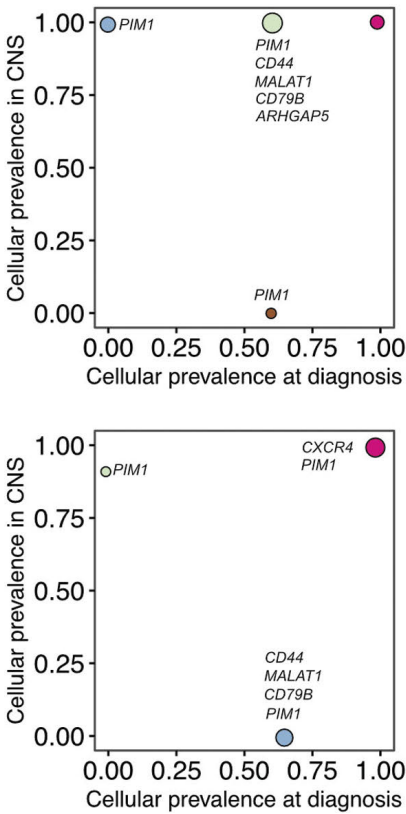
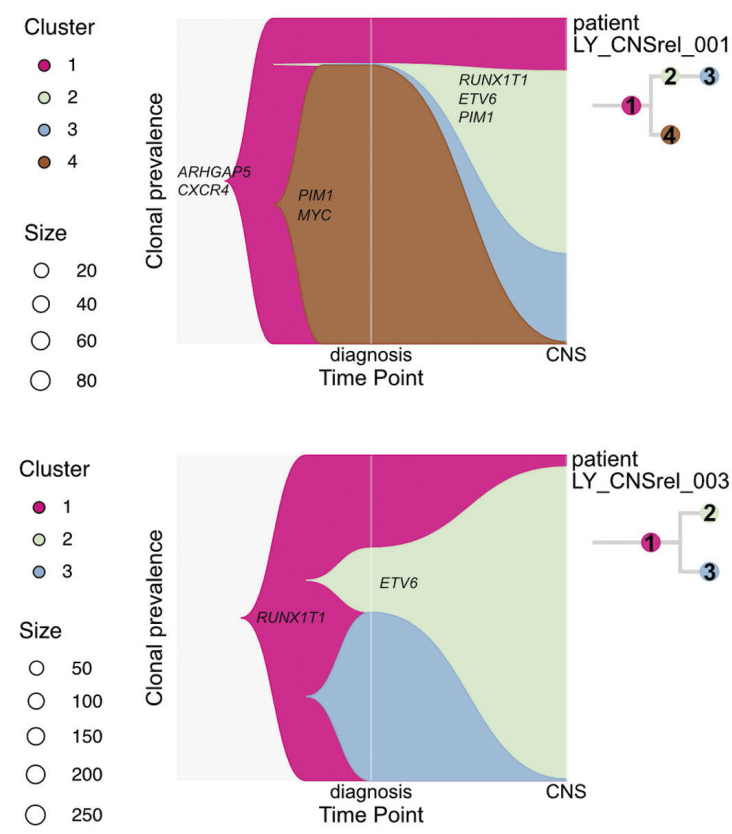

Cluster
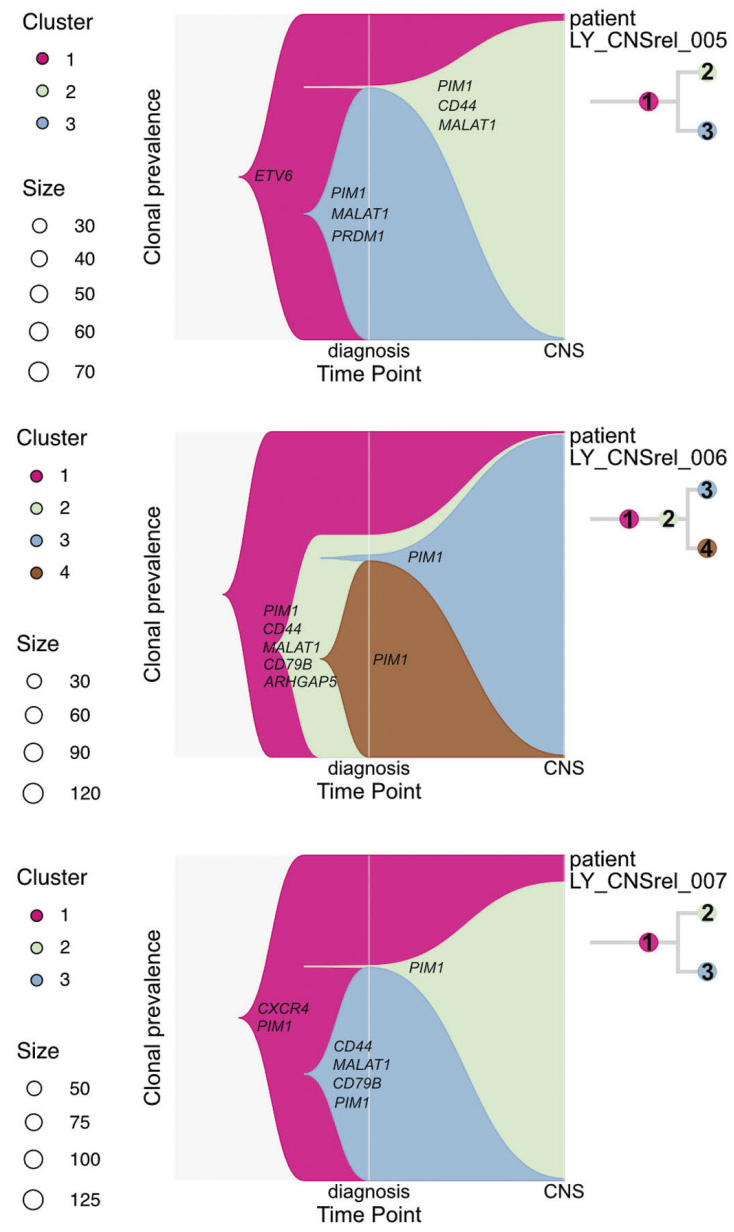

Figure 1. Clonal evolution patterns associated with central nervous system relapse. PyClone analysis to retrace clonal phylogenies in five cases with documented central nervous system (CNS) dissemination. The leftmost column shows variant allele frequencies (VAF) at diagnosis vs. VAF in CNS sample. The middle column depicts clusters of mutation cellular prevalence. The rightmost column shows time sweep plots that graphically illustrated clonal tides over the course of relapse. Representative diffuse large B-cell lymphoma genes are labeled. 
Proportion of samples with mutation Group difference
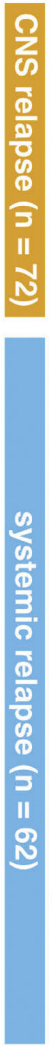

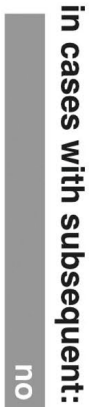

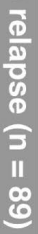

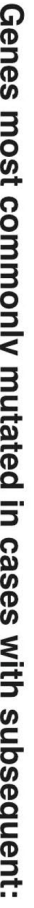

HIST$$
\text { SGK }
$$
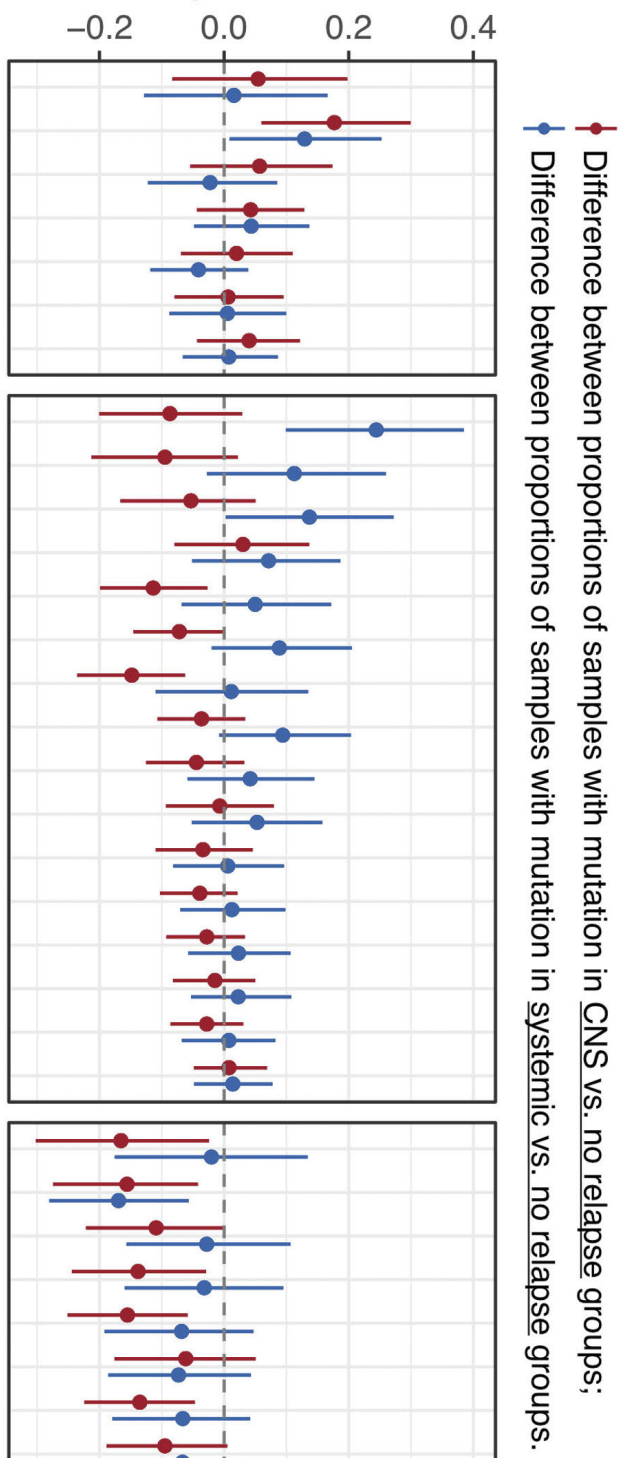

Figure 2. Gene mutations associated with central nervous system and systemic relapse. The barplot shows the proportion of samples harboring given gene mutations. Shown are 45 genes. The total sample size is 223 samples ( $n=72$ with central nervous system [CNS] relapse, $n=62$ with systemic relapse and $n=89$ without relapse). The line plot shows differences between proportions of mutated samples, when comparing either CNS vs. no relapse, or systemic vs. no relapse. The lines indicate the ranges of the credible intervals for group differences, and the small circles represent the point estimates of the group differences. 
A

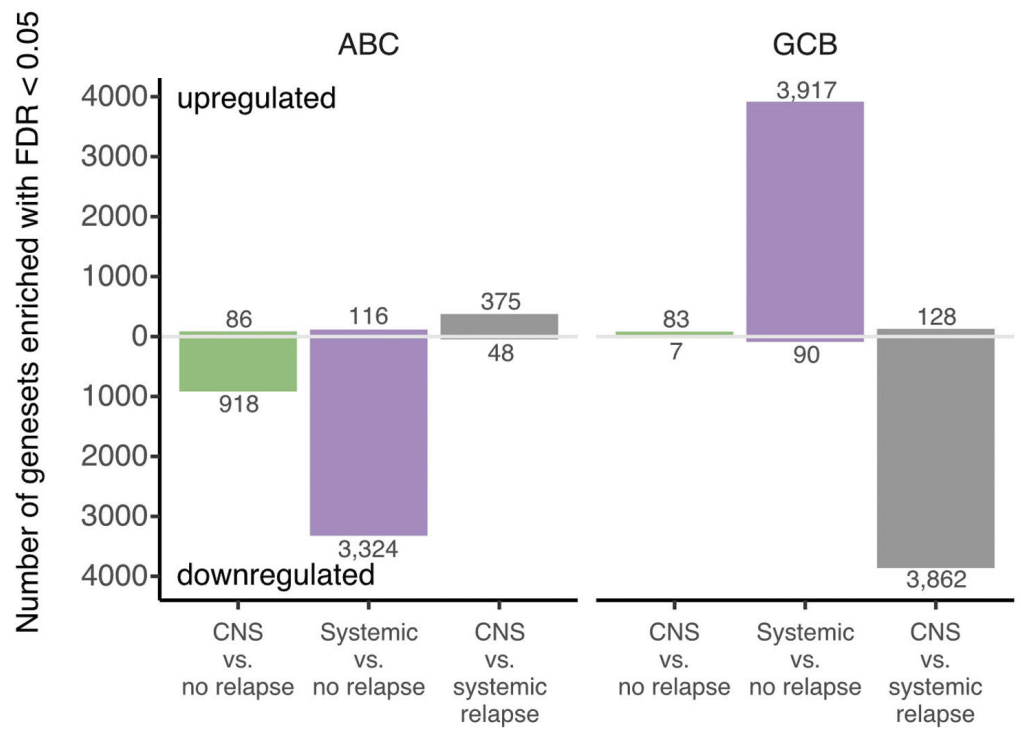

CNS vs. no relapse Systemic vs. no relapse CNS vs. systemic relapse

B

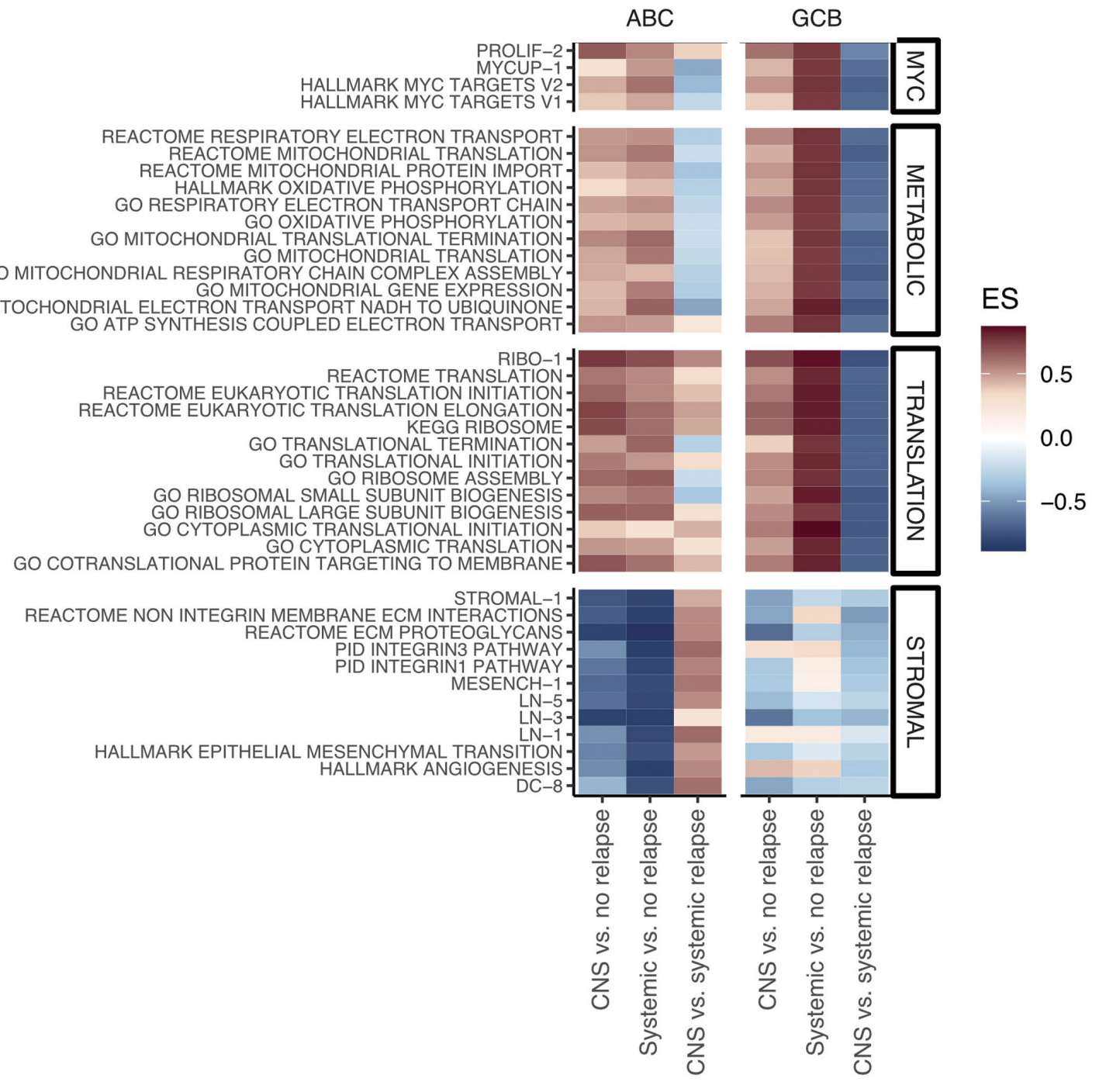

GO MITOCHONDRIAL TRANSLATION

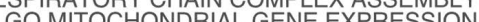
GO MITOCHONDRIAL ELECTRC

GO ATP SYNTHESIS COUPLEDELECTRON TRANSPORT

Figure 3. Pathways associated with central nervous system and systemic relapse. (A) Number of gene sets enriched by contrast and by cell-of-origin (COO), based on differential gene expression analysis. Only gene sets with adjusted $P$-value of $<0.05$ are shown. (B) Heatmap showing the enrichment scores (ES) of the most down- and upregulated pathways by contrast and by COO. Pathways are grouped by biological theme. 
ly additional CNS-specific processes not captured through transcriptome profiling.

Herein, we present a comprehensive description of clonal evolution, gene mutations and gene expression changes associated with CNS and systemic relapse in DLBCL. Our analysis of clonal evolution patterns underlying CNS relapse revealed clear clonal divergence. In general, mutations specifically associated with the relapsing clone were not found at measurable levels in preceding diagnostic specimens. Our cohort for exome sequencing of paired samples was limited due to inherent challenges associated with collecting biospecimens of this nature, as a limited number of patients undergo sampling of brain parenchyma at the time of relapse. Further, a divergent mode of clonal evolution is not unique to CNS relapse, but rather a general property of cancers that harbor underlying intra-tumoral heterogeneity. Divergent evolution has also been demonstrated in DLBCL, ${ }^{10,11}$ although - to the best of our knowledge no prior study has applied state-of-the-art phylogenetic reconstruction tools to trace clonal trajectories in the specific context of CNS relapse. The phylogenetic portraits that we could draw exert a cautionary tale, illustrating that efforts to improve patient outcomes will need to take into consideration the molecular landscape at the time of lymphoma relapse. Moreover, well-known challenges to predict CNS relapse may be explained by both inter-patient and intra-tumor heterogeneity that is universally recognized in DLBCL.

Alterations of $\mathrm{ABC}$ subtype-associated genes are frequently seen in primary CNS lymphoma, ${ }^{12}$ which is often of an $\mathrm{ABC}$ phenotype, ${ }^{13}$ and are also characteristic of the recently identified C5/MCD subtype of DLBCL that is associated with extranodal involvement and poor outcome. ${ }^{14,15}$ In the gene mutation analysis, the strongest enrichment was seen for $M Y D 88$, although this gene was almost as often mutated in cases with subsequent systemic relapse. Conversely, we found that several gene mutations that were more commonly seen in patients experiencing systemic, as opposed to CNS relapse (e.g., TP53). Other mutations such as KTM2D, characteristic of the C3/EZB subtype of DLBCL, appeared to be comparatively infrequent in CNS relapse cases, perhaps suggesting that the KTM2D-mutant phenotype is not conducive to establishing tumors in the CNS. Lastly, we found that biological pathways were differentially enriched between clinical risk groups in the $\mathrm{ABC}$ versus GCB subtype of DLBCL. Signals underlying CNS relapse were overall weaker compared with signals underlying systemic relapse, potentially reflective of clonal divergence and resulting phenotypic shifts that may accompany CNS relapse. It seems unlikely that a gene expression-based biomarker can be developed to positively identify patients at highest risk of CNS relapse, beyond the information that is already contained within the transcriptional footprints that define known DLBCL subtypes. In contrast, large-scale, integrative analyses and in-depth characterization of clonal trajectories hold the promise to increase our ability to understand dissemination of DLBCL into the CNS.

Keren Isaev, ${ }^{1}$ Daisuke Ennishi, ${ }^{2}$ Laura Hilton, ${ }^{3}$ Brian Skinnider, ${ }^{2}$ Karen L. Mungall, ${ }^{4}$ Andrew J. Mungall, ${ }^{4}$ Mehran Bakthiari, ${ }^{1}$ Rosemarie Tremblay-LeMay, Anjali Silva, ${ }^{1}$ Susana Ben-Neriah, ${ }^{2}$ Merrill Boyle, Diego Villa, ${ }^{2}$ Marco A. Marra, ${ }^{4}$ Christian Steidl, ${ }^{2}$ Randy D. Gascoyne, ${ }^{2}$ Ryan D. Morin, ${ }^{3}$ Kerry J. Savage, David W. Scott ${ }^{2 \#}$ and Robert Kridel ${ }^{1 \#}$

${ }^{1}$ Princess Margaret Cancer Center - University Health Network,
Toronto, Ontario; ${ }^{2}$ Center for Lymphoid Cancer, Britsh Columbia Cancer, Vancouver, British Columbia; ${ }^{3}$ Simon Fraser University, Burnaby, British Columbia; ${ }^{4}$ Canada's Michael Smith Genome Sciences Center, British Columbia Cancer, Vancouver, British Columbia and 5 Laboratory Medicine Program - University Health Network, Toronto, Ontario, Canada.

\#DWS and RK contributed equally as co-senior authors.

Correspondence:

ROBERTKRIDEL - robert.kride@@uhn.ca

doi:10.3324/haematol.2020.255950

Received: April 22, 2020.

Accepted: August 6, 2020.

Pre-published: August 13, 2020.

Disclosures: no conflicts of interest to disclose.

Contributions: RK and DWS designed the study, collected data, analyzed data and wrote the manuscript; KI analyzed data and wrote the manuscript. $D E, B S, M B, R T L, B M, S B N$, and $M B$ reviewed samples, extracted and inventoried samples and/or provided data; $L H$ and RM provided bioinformatic guidance; $K M$, $A J M$ and MAM provided critical support for exome sequencing and analysis; DV, CS, RDG and KJS provided samples and clinical data; $A S$ reviewed and corrected bioinformatic scripts related to this manuscript; all authors approved the final version of the manuscript.

Acknowledgments: we thank the expert staff of the Michael Smith's Genome Sciences Center at Britsh Columbia Cancer Center in Vancouver and The Center for Applied Genomics at SickKids in Toronto for generating exome sequencing data and gene expression. We thank Dr. Osvaldo

Espin-Garcia for expert statistical advice.

Funding: this work was supported by an Innovation Grant (award \# 703505) from the Canadian Cancer Society Research Institute (DWS), by a Genome Canada Large-Scale Applied Research Project (Genome Canada \#13124, Genome BC \#271LYM, Canadian Institutes of Health Research \#GP1-155873 and the BC Cancer Foundation) (CS, DWS, MAM), the Ontario

Research Fund (RK), and by the Princess Margaret Cancer

Foundation (RK).

\section{References}

1. Kridel R, Dietrich P-Y. Prevention of CNS relapse in diffuse large B-cell lymphoma. Lancet Oncol. 2011;12(13):1258-1266.

2. Boehme V, Schmitz N, Zeynalova S, Loeffler M, Pfreundschuh M. CNS events in elderly patients with aggressive lymphoma treated with modern chemotherapy (CHOP-14) with or without rituximab: an analysis of patients treated in the RICOVER-60 trial of the German High-Grade Non-Hodgkin Lymphoma Study Group (DSHNHL). Blood. 2009;113(17):3896-3902.

3. Schmitz N, Zeynalova S, Nickelsen M, et al. CNS International Prognostic Index: a risk model for CNS relapse in patients with diffuse large B-cell lymphoma treated with R-CHOP. J Clin Oncol. 2016:34(26):3150-3156.

4. Boehme V, Zeynalova S, Kloess M, et al. Incidence and risk factors of central nervous system recurrence in aggressive lymphoma--a survey of 1693 patients treated in protocols of the German HighGrade Non-Hodgkin's Lymphoma Study Group (DSHNHL). Ann Oncol. 2007;18(1):149-157

5. El-Galaly TC, Villa D, Michaelsen TY, et al. The number of extranodal sites assessed by PET/CT scan is a powerful predictor of CNS relapse for patients with diffuse large B-cell lymphoma: an international multicenter study of 1532 patients treated with chemoimmunotherapy. Eur J Cancer. 2017;75:195-203

6. Savage KJ, Slack GW, Mottok A, et al. Impact of dual expression of MYC and BCL2 by immunohistochemistry on the risk of CNS relapse in DLBCL. Blood. 2016;127(18):2182-2188.

7. Klanova M, Sehn LH, Bence-Bruckler I, et al. Integration of cell of origin into the clinical CNS International Prognostic Index improves CNS relapse prediction in DLBCL. Blood. 2019;133(9): 919-926. 
8. Pasqualucci L, Neumeister P, Goossens T, et al. Hypermutation of multiple proto-oncogenes in B-cell diffuse large-cell lymphomas. Nature. 2001;412(6844):341-346.

9. Ennishi D, Jiang A, Boyle M, et al. Double-hit gene expression signature defines a distinct subgroup of germinal center B-cell-like diffuse large B-Cell lymphoma. J Clin Oncol. 2019;37(3):190-201.

10. Juskevicius D, Lorber T, Gsponer J, et al. Distinct genetic evolution patterns of relapsing diffuse large B-cell lymphoma revealed by genome-wide copy number aberration and targeted sequencing analysis. Leukemia. 2016;30(12):2385-2395.

11. Greenawalt DM, Liang WS, Saif S, et al. Comparative analysis of primary versus relapse/refractory DLBCL identifies shifts in mutation spectrum. Oncotarget. 2017;8(59):99237-99244.
12. Lesokhin AM, Ansell SM, Armand P, et al. Nivolumab in patients with relapsed or refractory hematologic malignancy: Preliminary results of a phase ib study. J Clin Oncol. 2016;34(23):2698-2704.

13. Booman M, Douwes J, Glas AM, et al. Primary testicular diffuse large B-cell lymphomas have activated B-cell-like subtype characteristics. J Pathol. 2006;210(2):163-171

14. Chapuy B, Stewart C, Dunford AJ, et al. Molecular subtypes of diffuse large $\mathrm{B}$ cell lymphoma are associated with distinct pathogenic mechanisms and outcomes. Nat Med. 2018;24(5):679-690.

15. Schmitz R, Wright GW, Huang DW, et al. Genetics and pathogenesis of diffuse large B-cell lymphoma. N Engl J Med. 2018; 378(15):1396-1407. 